\section{Assoziation zwischen ADHS und Asthma}

\section{Wissenschaftler aus Schweden wollten die Beziehung zwischen Asthma und ADHS erforschen. Anhand der Untersuchung von mehreren hundert Zwillingspaaren konnten sie einen Zusammenhang feststellen.}

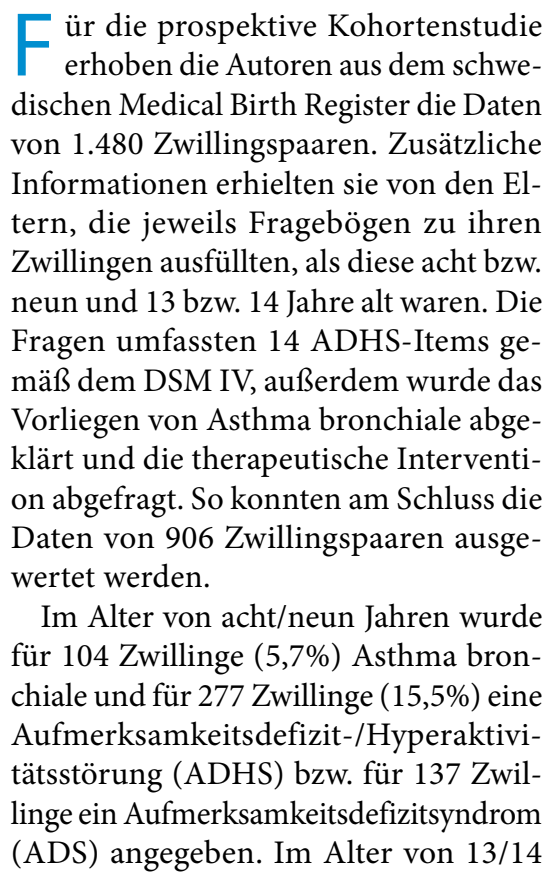

Jahren wurden 138 Zwillinge (7,7\%) mit Asthma und 128 Zwillinge $(7,1 \%)$ mit ADHS bzw. 125 (6,9\% ) mit ADS registriert.

Asthma im Alter von acht/neun Jahren ging mit einem zweifach erhöhtem Risiko für ADHS, nicht aber für ADS im Alter von acht/neun und 13/14 Jahren einher. Das Vorliegen einer Neurodermitis zeigte dagegen keinen Zusammenhang mit einer ADHS. Auch die Anwendung einer Asthmamedikation beeinflusste das Risiko für eine ADHS nicht. Jungen waren häufiger von der Assoziation Asthma/ADS betroffen. Der sozioökonomische Status korrelierte mit dem Auftreten von ADHS/ADS, führte jedoch nicht zu einem erhöhten Auftreten der Assoziation Asthma/ADHS. Die genetischen Untersuchungen lassen darauf schließen, dass eine genetische Überlappung zwischen Asthma und ADHS/ADS besteht.
Morgensen $\mathrm{N}$ et al. Association between childhood asthma and ADHD symptoms in adolescence - a prospective populations-based twin study. Allergy 2011: 66: 1224-30

Kommentar: Was klinisch schon lange vermutet wurde, wird jetzt durch eine Studie bestätigt: Es besteht eine Assoziation zwischen Asthma und ADHS - unabhängig von der Medikation und auch nicht hervorgerufen durch eine zusätzlich bestehende Neurodermitis mit einer kausalen Beziehung zwischen starkem Pruritus und motorischer Unruhe.

Interessant ist auch die Beobachtung, dass diese Assoziation überwiegend zwischen der kombinierten Form der ADHS und nicht der überwiegend unaufmerksamen Form auftritt. Dies lässt darauf schließen, dass es sich hier um genetisch unterschiedliche Subtypen handelt. Die Aussagekraft der Studie wird jedoch durch die Methodik eingeschränkt: Die Erkrankungen Asthma bzw. ADHS/ADS wurden nur durch Befragungen der Eltern diagnostiziert. Dessen ungeachtet sollten bei Kindern mit Asthma darauf geachtet werden, ob zusätzlich eine ADHS besteht.

Dr. Kirsten Stollhoff

\title{
Gefährden Kernkraftwerke Kinderleben?
}

Es gibt sehr widersprüchliche Studien zu dem Thema, ob Kinder, die in der Nähe eines Kernkraftwerkes aufwachsen, ein erhöhtes Krebsrisiko haben. In einer aktuellen Kohortenstudie aus der Schweiz wurde kein erhöhtes Risiko nachgewiesen.

D aten des Schweizerische Krebsregisters zeigten, dass zwischen dem 1. Januar 1985 und dem 31. Dezember 2009 bei 4.180 Kindern (Alter 0-15 Jahre) Krebs diagnostiziert wurde. Bei 4.090 dieser Kinder konnte der Wohnort zum Zeitpunkt der Diagnose und bei 2.925 der Wohnort zum Zeitpunkt der Geburt festgestellt werden. So konnte errechnet werden, ob die Kinder zum Zeitpunkt der Diagnose/Geburt in weniger als $5 \mathrm{~km}$ Entfernung, in 5-15 km Entfernung oder in mehr als $15 \mathrm{~km}$ von einem Atomkraftwerk oder anderen nuklearen Einrichtungen entfernt aufgewachsen/geboren worden waren.

Lediglich die Leukämieraten waren im Einzugsgebiet der AKWs leicht, aber nicht signifikant erhöht.
Spycher BD et al. Childhood cancer and nuclear power plants in Switzerland: a census-based cohort study. Int J Epidemiol 2011; July 27

[Epub ahead of print]

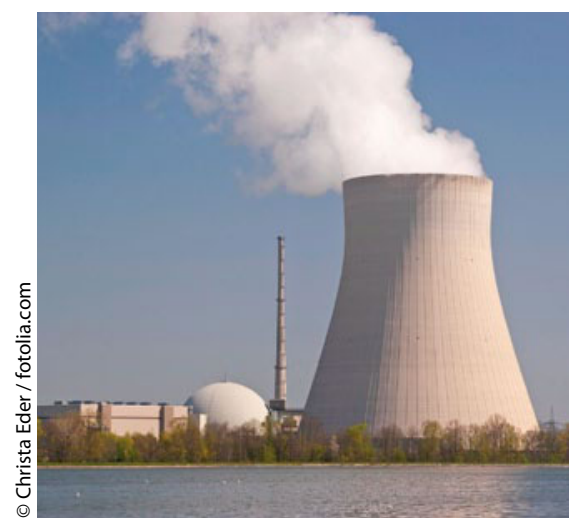

Kommentar: Diese große, umfangreiche Kohortenstudie konnte also keinen Beweis für einen Zusammenhang zwischen einem Wohnort in der Nähe eines AKWs und dem Risiko, an Krebs zu erkranken, belegen. Die gefundene geringe Abweichungen bei der Leukämie sind nach Angaben der Forscher noch als rein zufällig anzusehen. Eine 2007 veröffentlichte Studie aus Deutschland zeigte hingegen ein erhöhtes Krebsrisiko bei Kindern, die im Umkreis von fünf Kilometern von Kernkraftwerken wohnen. Das Risiko war insbesondere für Leukämie bei Kleinkindern erhöht. Was aber letztlich die Erkrankung auslöst, war in der vom Bundesamt für Strahlenschutz in Auftrag gegebenen Studie offen geblieben. Rund ein Prozent der Schweizer Bevölkerung lebt im Umkreis von fünf Kilometern und $10 \%$ im Umkreis von 15 Kilometern eines AKWs. Auch die Schweiz plant den Ausstieg aus der Atomkraft, und zwar bis 2019.

Prof. Dr. Kurt Malberg 